and Hideki Yukawa's wonderful paper of 1935 in which he introduced the idea of virtual quanta, proposed the existence of a heavy nuclear force quantum, and prefigured today's Standard Model of beta-decay.

Tomonaga's style is informal and often humorous. In the epilogue, he thinks of "an old sailor talking on and on about his youthful adventures" as he "spins a yarn". The story of those 20-odd years in physics has been told before, but never from quite this perspective. Thanks to Oka's translation, Tomonaga's lectures will now be read and enjoyed by the wide audience they deserve.

Ian Aitchison is in the Department of Theoretical Physics, University of Oxford, 1 Keble Road, Oxford OX1 3NP, UK.

\section{Plant roots}

\section{The Origin and Early Diversification of Land Plants: A Cladistic Study}

by Paul Kenrick and Peter R. Crane

Smithsonian Institution Press: 1997. Pp. 592.

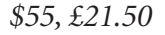

\section{Sandy Knapp}

The evolution and diversification of life has always held a great fascination for biologists. If we are to understand the patterns of relationship of life today, it is imperative that we have some understanding of those in the past; it puts our small branches in perspective. The extremes of innovation and diversification that have occurred throughout history tell compelling stories, but need to be put into clear systematic perspective to be analysed effectively.

For plants, the emergence of terrestrial forms was the start of a period of unparalleled diversification and innovation. The pivotal events leading to the origins and diversification of land plants are similar to those involved in the Cambrian 'explosion' that so influenced the diversification of the Metazoa. The metazoan fossils of the Burgess Shale endlessly fascinate both scientists and the general public, but the plant fossils of the Rhynie chert are just as fascinating from the evolutionary point of view.

The study of evolution depends on phylogeny: without hypotheses of the patterns of character distribution, and thus relationships between groups of organisms, no evolutionary hypotheses can really be constructed. The power and beauty of the approach of Paul Kenrick and Peter R. Crane to the analysis of land-plant relationships lies in their use of cladistic methodology. The focus on comparative morphology, and the testing of their own and other hypotheses of specific relationships, means that they truly achieve the objective set out in the first pages of the book: "to begin to synthesize the data that are currently available for resolving phylogenetic, and hence evolutionary, patterns

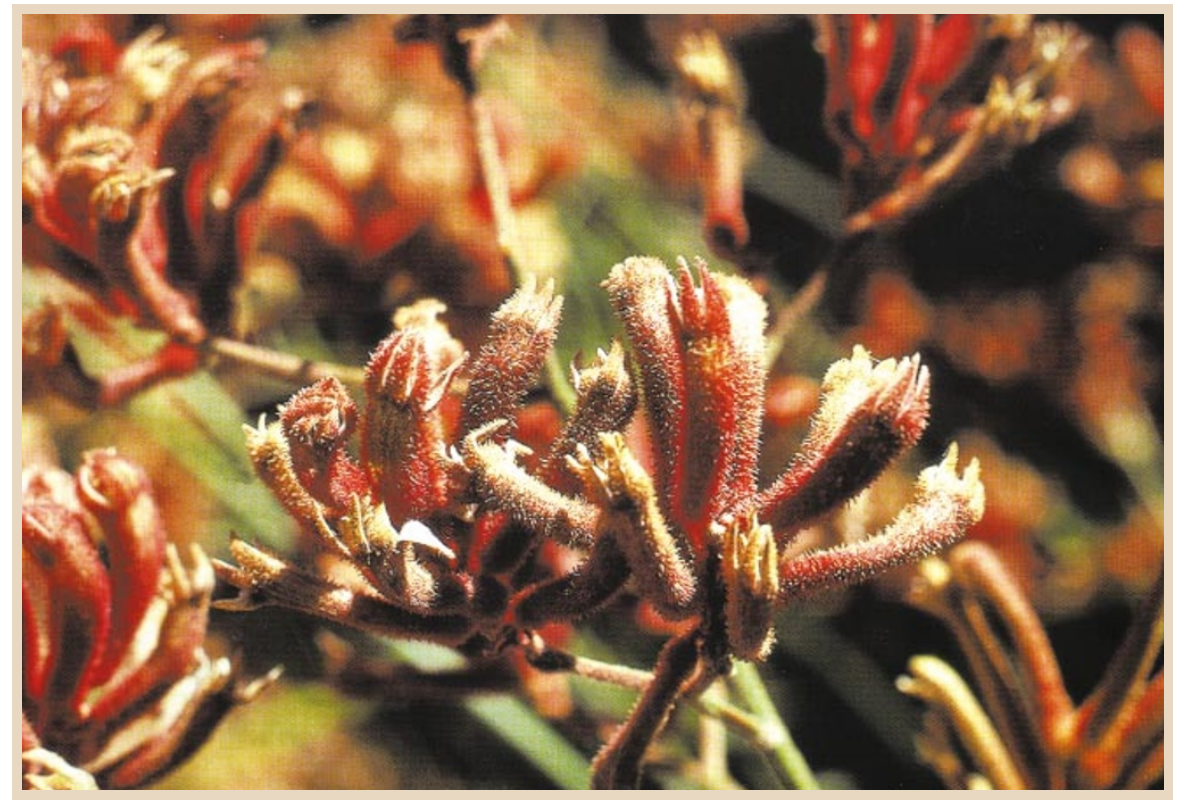

\title{
Colourful explanations
}

The island continent of Australia is known for its bizarre, colourful and often unique wildlife, from the marsupials and egg-laying mammals to the sea creatures of the Great Barrier Reef. Western Australia is particularly noted for its wildflowers, with more than 2,000 native species including the official state flower, the kangaroo

among 'basal' groups of land plants."

This is not a book that tells a story based on an existing phylogeny; these are the real data, the characters that resolve the pattern, warts and all. It is tempting to think that evolution can be studied by simply plotting characters of interest on pre-existing phylogenies (usually constructed using molecular data), but Kenrick and Crane show how truly effective the alternative approach can be: that of an in-depth analysis of characters and their distribution using cladistic methodologies.

The book is constructed in a nesting set of detailed treatments of ever more detailed monophyletic groups. For example, the chapter on the relationships between embryophytes is followed by a chapter on the relationships among the polysporangiophytes, tracheophytes and euphyllophytes, all members of the preceding group. Each chapter is constructed similarly, making the characters and methodology comparable at each stage of the analysis. The choice of taxa used in each analysis is detailed, and all characters used in the analysis are described in detail and their coding justified. This is unusual in a book of this sort, and is a welcome innovation. Kenrick and Crane also analyse carefully the degree of applicability of each character and state how many taxa had missing data for each character used.

This may seem like unnecessary detail for a synthetic book of this kind, but it allows one to assess the authors' confidence in a paw (above). In A Natural History of Australia (Academic Press, \$44.95), Tim M. Berra sets out to explain how the flora and fauna have been shaped by the isolation and aridity of the continent. The book is aimed at the interested visitor, incorporating a geography of Australia and useful information for the traveller.

character, rather than just taking their word for it. Characters with significant missing data - often those cellular details that are difficult to see in fossils - are also discussed in detail, and other potentially relevant characters not used in the analysis are considered as well. This honest and open approach, not often used because it reveals the limits of both the data and those analysing it, will surely stimulate palaeobotanists to reexamine fossils in museums around the world to redefine and refine the knowledge of these potentially useful characters.

The cladistic classification of the plant kingdom (Chlorobionta) is a model for how monophyly can be used as the defining principle for classification. A table of synapomorphy-based definitions of monophyletic higher taxa provides an extremely effective summary of the power of the cladistic approach. The in-depth discussion of developmental transformations in the context of taxic homology puts the characters used in Kenrick and Crane's analyses in perspective and allows them to set the stage for the next advances in the understanding of land-plant evolution.

This excellent and detailed book stands as a model for how to approach the study of evolution, and is an essential addition to the bookshelves of anyone interested in the scope and diversification of life.

Sandy Knapp is in the Department of Botany, Natural History Museum, Cromwell Road, London SW7 5BD, UK. 